\title{
L'administration camerounaise dépouillée de ses prérogatives de puissance publique devant le juge judiciaire
}

Regard sur la jurisprudence en matière de voie de fait administrative The Cameroon administration stripped of its prerogatives of public power before the ordinary court. An examination of case-law on administrative trespass

Jacques Bipele Kemfouedio

\section{(2) OpenEdition}

Édition électronique

URL : http://journals.openedition.org/add/939

DOI : $10.4000 /$ add. 939

ISSN : 2606-1988

\section{Éditeur}

Presses universitaires de Rouen et du Havre

Édition imprimée

Pagination : $31-59$

ISBN : 979-10-240-0937-7

ISSN : 1955-0855

\section{Référence électronique}

Jacques Bipele Kemfouedio, «L'administration camerounaise dépouillée de ses prérogatives de puissance publique devant le juge judiciaire », Les Annales de droit [En ligne], 12 | 2018, mis en ligne le 05 février 2019, consulté le 05 décembre 2019. URL : http://journals.openedition.org/add/939; DOI : 10.4000/add. 939 


\title{
L'administration camerounaise dépouillée de ses prérogatives de puissance publique devant le juge judiciaire
}

\author{
Regard sur la jurisprudence \\ en matière de voie de fait administrative
}

Jacques BIPELE KeMFouedio

La voie de fait administrative fait partir des sources intarissables qui continuent d'alimenter les débats dans le champ scientifique ${ }^{1}$. L'arrêt

1. La voie de fait administrative s'analyse comme une théorie d'origine jurisprudentielle protectrice des droits des administrés en ce qu'elle entraîne pour l'administration la perte de la majeure partie de ses privilèges traditionnels. Elle serait constituée au Cameroun si l'administration accomplit un acte matériel représentant une irrégularité manifeste, soit parce qu'elle exécute une décision ne se rattachant pas à un pouvoir qui lui appartient comme une décision grossièrement illégale ou annulée par une juridiction, soit parce qu'elle exécute, selon une procédure grossièrement illégale, une décision même légale, et à condition que cet agissement porte atteinte à la propriété mobilière, immobilière ou à une liberté publique (Serge Guinchard et Thierry Debard, Lexique des termes juridiques, Paris, Dalloz, 2015, p. 1074-1075). Maurice Hauriou définit la voie de fait en la découpant en deux catégories: voie de fait par manque de procédure et voie de fait par manque de droit (Précis de droit administratif et de droit public, Paris, Sirey, 1927, p. 30). Pour l'auteur, la voie de fait par manque de procédure s'observe lorsque l'administration procède à l'exécution forcée d'un acte administratif en dehors des cas où celle-ci est possible. Par contre, il $\mathrm{y}$ a voie de fait par manque de droit dans l'hypothèse constitutive d'une irrégularité totale, où l'administration exécute une opération matérielle dépourvue de tout fondement juridique. En effet, elle use de la contrainte matérielle à l'encontre d'un particulier pour assurer l'exécution d'une de ses volontés, alors qu'aucun texte, aucun principe de droit ou aucun acte juridique pris en vertu d'un texte ne lui permettait de constituer cette volonté en un titre juridique. Il y a alors ici non seulement irrégularité de la voie de fait par manque de procédure, mais s'y ajoute l'absence même de droit sur le plan du fond (Carl Irani, La compétence judiciaire en matière administrative en droit libanais et en droit français, thèse de doctorat, Université de Grenoble Alpes, 2014, p. 84 et suiv.). Au regard de cette analyse, deux conditions cumulatives doivent être remplies au Cameroun pour que la voie de fait existe. La première est l'exécution matérielle grossière. La doctrine ainsi que la jurisprudence affirment ce principe depuis toujours (T. confl., 13 déc. 1947, Hilaire D., 1948, 
Bergoend du tribunal des conflits français rendu le 17 juin 2013 vient d'ailleurs confirmer non seulement le caractère actuel de ces débats, mais aussi l'aspect évolutif de la notion ${ }^{2}$. Il s'agit de l'un des rares domaines dont le contentieux conduit à traiter l'administration comme si elle n'était qu'un simple particulier. C'est une évidence au Cameroun, car le contentieux de la voie de fait, qui implique inévitablement l'autorité administrative, relève en principe de la compétence du juge judiciaire. Ce type de contentieux s'inscrit ainsi en marge de la logique du principe de la séparation des autorités administratives et judiciaires, car, bien que

I-62 et CE, 15 mars 1957, Deloffre, Rec. 75). Le doyen Georges Vedel déclare d'ailleurs que "la voie de fait est toujours constituée par une opération administrative, jamais par une pure décision indépendante de toutes mesures d'exécution» ( $\mathrm{La}$ juridiction administrative compétente pour prévenir, faire cesser ou réparer la voie de fait administrative», JCP 1950, I-851). La seconde condition est l'absence de fondement juridique de l'exécution matérielle ou la présence d'une irrégularité grave. En compulsant la jurisprudence administrative camerounaise, le juge définit la voie de fait comme des "actes tellement irréguliers qu'ils perdent tout caractère administratif» (CFJ/SCAY, arrêt $n^{\circ}$ 20, 4 nov. 1966, Max Keller Ndongo c/ État du Cameroun). Cette définition est reprise dans l'affaire Nomeny Nguissi Émile c/ État du Cameroun (CS/CA, jugement $n^{\circ} 63,26$ mai 1988). Dans les affaires Yikam Jérémie et Kuela Valérie Norbert, le juge définit la voie de fait comme un « acte ou agissement de l'administration dont l'irrégularité porte une atteinte grave à un droit fondamental de la personne» (CS/CA, jugement $\mathrm{n}^{\circ}$ 31/ADD/04-05, 29 déc. 2004, Yikam Jérémie c/ État du Cameroun; CS/AP, arrêt $n^{0}$ 07/A, 16 déc. 2004, Kuela Valérie Norbert (sous-préfet d'Akonolinga) et Minlo Auguste (adjoint d'arrondissement) c/ Eben Édouard Stève (commandant de brigade de cette localité). Quoi qu'il en soit, pour que cette qualification juridique soit retenue, l'autorité administrative doit avoir agi dans l'extrême arbitraire en s'affranchissant complètement du droit de sorte que l'acte juridique pris ou l'opération matérielle accomplie se trouve « dépouillé de tout caractère administratif » (Cass. civ, 27 fév. 1950, Maire, JCP G, 1950 II, p. 5517, note R. Cavaroc).

2. En effet, depuis l'intervention de l'arrêt Bergoend du 17 juin 2013, la notion de voie de fait a évolué en France. Pour le Tribunal des conflits, «il n’y a voie de fait de la part de l'administration, justifiant, par exception au principe de séparation des autorités administratives et judiciaires, la compétence des juridictions de l'ordre judiciaire pour en ordonner la cessation et la réparation, que dans la mesure où l'administration, soit a procédé à l'exécution forcée, dans des conditions irrégulières, d'une décision, même régulière, portant atteinte à la liberté individuelle ou aboutissant à l'extinction d'un droit de propriété, soit a pris une décision qui a les mêmes effets d'atteinte à la liberté individuelle ou l'extinction d'un droit de propriété et qui est manifestement insusceptible d'être rattachée à un pouvoir appartenant à l'autorité administrative ». Avant cette définition, la voie de fait s'entendait comme une «atteinte grave à la propriété privée ou à une liberté fondamentale» (T. confl., 19 nov. 2001, $\mathrm{M}^{\mathrm{lle}}$ Mohamed). Sur l'évolution de la voie de fait, lire Julie Cornu, «Les métamorphoses de la voie de fait : changements attendus et perspectives », RDP 2017 , p. 571 et suiv. 
l'administration soit impliquée dans le litige, c'est le juge judiciaire qui est compétent pour statuer sur l'affaire ${ }^{3}$.

L'administration se rapporte à des activités d'un type particulier, caractérisées par les idées de souveraineté, de commandement, d'autorité, et tendant à la satisfaction prioritaire de l'intérêt général. Ces activités sont placées pour cette raison sous un régime de puissance publique qui, à tout moment et en tout lieu, permet de l'imposer aux intérêts des particuliers grâce à des prérogatives sans équivalent en droit privé. Ainsi, l'administration est le bras séculier de l'État. Elle doit à cet effet accomplir diverses missions qui sont variables dans leur nature et qui ont un même dessein: la satisfaction des besoins d'intérêt général tout en se soumettant aux exigences légales.

Le principe de légalité permet dès lors de soumettre l'action administrative à la règle de droit. Cette soumission, garantie par le juge, permet de préserver les droits des particuliers contre l'arbitraire de l'administration ${ }^{4}$. En vertu du principe de la séparation des autorités administrative et judiciaire, c'est le juge administratif, juge de droit

3. L'interdiction faite aux tribunaux judiciaires de connaître des agissements de l'administration est antérieure à la Révolution. On la fait remonter généralement à l'édit de Saint-Germain-en-Laye de février 1641. Elle fut solennellement proclamée par la loi des 16-24 août 1790 et le décret du 16 fructidor an III. Selon cette loi, «les fonctions judiciaires sont et demeurent toujours séparées des fonctions administratives». «Les juges ne pourront, à peine de forfaiture, troubler de quelque manière que ce soit les opérations des corps administratifs, ni citer devant eux des administrateurs pour raison de leurs fonctions. " L'administration se définit, d'un premier point de vue, non seulement comme un ensemble d'autorités et d'agents qui appartiennent au secteur public et agissent pour le compte des différents organismes et collectivités publics, mais aussi comme les différents services que constituent ces organismes et par lesquels sont développées leurs interventions. D’un second point de vue, ce terme se rapporte à des activités d'un type particulier, caractérisées par les idées de souveraineté, de commande, d'autorité, et tendant à la satisfaction prioritaire de l'intérêt général (Martin Akendengue, "Le principe de l'égalité des parties en procédure contentieuse administrative au Gabon », dans Emmanuel Decaux (dir.), Justice et droits de l'homme, Bruxelles, Bruylant, 2003, p. 366-367).

4. Pour Maurice Kamto, «la justice administrative est l'expression concrète de la protection des citoyens contre les risques d'arbitraire de l'administration ». "Elle apparaît en effet comme un moyen de défense de l'individu contre les abus du pouvoir, non pas en vue de compromettre l'autorité de celui-ci, mais pour lutter contre d'éventuelles dérives despotiques" (Droit administratif processuel du Cameroun. Que faire en cas de litige avec l'administration? Yaoundé, Presses universitaires du Cameroun, 1990, p. 7 ; voir aussi Samson Dossoumon, «Réflexions sur le contrôle juridictionnel de l'administration dans les pays en voie de développement d'Afrique noire francophone", Revue béninoise des sciences juridiques et administratives, $\mathrm{n}^{\mathrm{o}} 5$, 1985, p. 4 ; Georges Burdeau, Libertés publiques, Paris, LGDJ, 1961, p. 56. 
commun de l'administration ${ }^{5}$, qui est principalement compétent pour connaître des litiges dans lesquels l'administration est impliquée ${ }^{6}$. Ce principe consacré en France depuis longtemps a eu, du fait de la contagion coloniale, une affirmation non systématique au Cameroun, ceci à la faveur des dispositions pénales ${ }^{7}$ et des déclarations de la jurisprudence administrative $^{8}$. Ce principe consacre la répartition des compétences entre le juge administratif et le juge judiciaire ${ }^{9}$. Le noyau dur de cette répartition des compétences est basé sur la nature des matières en cause. Si les matières visées concernent le droit public, c'est la juridiction

5. Aux termes de l'article 14 de la loi camerounaise $n^{0} 2006 / 022$ du 29 décembre 2006 fixant l'organisation et le fonctionnement des tribunaux administratifs, «les tribunaux administratifs sont, sauf dispositions contraires de la loi, juges de droit commun du contentieux administratif en premier ressort».

6. Lire Bernard Pacteau, Manuel de contentieux administratif, Paris, PUF, 2006, p. 15 et suiv.; Olivier Gohin, Contentieux administratif, Pays LexisNexis, 2014, p. 59 et suiv; Joseph Owona, Le contentieux administratif de la République du Cameroun, Paris, L'Harmattan, 2011, p. 35 et suiv.

7. L. $\mathrm{n}^{\mathrm{o}}$ 2016/007, 12 juil. 2016, art. 126, portant Code pénal: «Est puni d'un emprisonnement de six (06) mois à cinq (05) ans : - le représentant de l'autorité exécutive qui intime des ordres ou des défenses à des Cours ou Tribunaux; - le magistrat qui intime des ordres ou des défenses à des autorités exécutives ou administratives. »

8. Dans l'affaire $\mathrm{M}^{\mathrm{me}}$ Aoua Hadja $\mathrm{c} /$ État du Cameroun (CS/CA, jugement $\mathrm{n}^{\circ}$ 213, 18 août 1972), le juge administratif affirme que «le principe de la séparation des pouvoirs interdit au juge administratif de statuer sur les actions qui mettent en cause le fonctionnement des services judiciaires ». Dans l'espèce Deffo c/ État du Cameroun (CS/CA, jugement $\mathrm{n}^{\circ}$ 59, 23 mars 2011), le juge administratif affirme qu' " au risque de violer le principe de la séparation des pouvoirs judiciaire et administratif, le juge ne saurait donner des injonctions à l'administration». Dans le cas Davide Njini Borno c/ État du Cameroun (CS/CA, jugement $n^{\circ} 71,3$ févr. 2010), le juge administratif camerounais justifie également l'interdiction de donner des injonctions par la «violation du principe de la séparation des pouvoirs entre le pouvoir judiciaire et l'administration». La consécration jurisprudentielle du principe de la séparation des autorités administrative et judiciaire se retrouve aussi dans les espèces Mfoumou Jean-Baptiste c/ État du Cameroun (CFJ/AP, arrêt $\mathrm{n}^{\circ}$ 17, 16 mars 1967), Tagny Mathieu c/ État du Cameroun (CFJ/AP, arrêt n ${ }^{\circ}$ 19, 16 mars 1967), Yombi Alphonse Bernard c/ État du Cameroun (CS/CA, jugement $n^{\circ}$ 44, 26 juin 1980), Mondoubou Théodore c/ État du Cameroun (CS/CA, jugement $n^{\circ} 43,26$ juin 1980), etc. Dans les deux derniers cas, le juge affirme: «Attendu que le principe de la séparation des pouvoirs administratif et juridictionnel et de la séparation des ordres juridictionnels, qui eut pour conséquence d'interdire aux tribunaux judiciaires de connaître des litiges nés de l'activité administrative, a également pour corollaire de soustraire à la connaissance du juge administratif tous les actes participant à l'exercice de la fonction judiciaire.»

9. Sur la séparation des compétences entre le juge administratif et le juge de droit commun, lire Célestin Keutcha Tchapnga, Précis de contentieux administratif au Cameroun: aspects de l'évolution récente, Paris, L'Harmattan, 2013, p. 39 et suiv. 
administrative qui est en principe compétente. Si, par contre, les matières en question relèvent du droit privé, c'est le juge judiciaire qui est compétent ${ }^{10}$. Le contentieux de la voie de fait administrative semble s'inscrire en marge de cette logique.

Comment s'appréhende à la lumière de la jurisprudence judiciaire en matière de voie de fait administrative le dépouillement de l'administration de ses prérogatives de puissance publique au Cameroun?

La question est particulièrement sensible dans un contexte où l'autorité administrative pense bénéficier d'une certaine immunité juridictionnelle la plaçant hors de portée de la justice pour citoyen ordinaire. Ce genre de comportement persiste aujourd'hui dans un contexte où le réflexe de domination autoritaire de certains fonctionnaires apparait comme un "atavisme d'une culture étatique d'un autre âge ${ }^{11}$ ». Le fonctionnaire camerounais comprend difficilement ou ne comprend pas du tout comment et pourquoi il peut être traduit devant le juge judiciaire en raison des actes qu'il a posés dans l'exercice de ses fonctions et, pense-t-il, dans l'intérêt général. Ce fonctionnaire, surtout lorsqu'il est autorité administrative (sous-préfet, préfet, gouverneur, ministre...), refuse souvent de répondre à la convocation du juge judiciaire qu'il qualifie souvent de "petit juge ». C'est donc dans un climat parfois tendu que s'analysent les relations entre l'administration et le juge judiciaire.

L'administration semble hostile au contrôle du juge judiciaire. Pourtant, dans un État de droit, cadre d'expression des droits et libertés individuelles et collectives ${ }^{12}$, la soumission de l'État et de ses démembrements à la règle de droit ne se discute pas. L'assurance pour tout citoyen que justice lui sera rendue en cas de violation par l'administration, de ses droits et libertés est un des fondements de l'État de droit. À l'examen de la jurisprudence judiciaire, on s'aperçoit que l'administration au Cameroun peut faire l'objet des poursuites devant le juge judiciaire lorsque l'autorité dans l'exercice de ses fonctions a posé des actes grossièrement illégaux : c'est la traduction de l'administration devant le juge judiciaire (1). Après instruction du dossier, le juge judiciaire, s'il estime que les faits sont établis, pourrait prononcer des sanctions à l'encontre de

10. Carl Irani, op. cit., p. 27 et suiv.

11. Maurice Kamto, "La mise en cause des autorités administratives... », art. cité, p. 336.

12. Sur ce point, on peut lire Gérard Conac, «Le juge et la construction de l'État de droit en Afrique francophone », dans L'État de droit. Mélanges en l'honneur de Guy Braibant, Paris, Dalloz, 1996, p. 47-55; Alban Coulibaly, «La rénovation de la justice en Afrique : le rôle du juge dans la construction de l'État de droit », Revue juridique et politique, $\mathrm{n}^{\mathrm{o}} 1,1999$, p. 50-66. 
l'administration défaillante: c'est la condamnation de l'administration devant le juge judiciaire (2).

\section{La traduction de l'administration devant le juge judiciaire}

En matière de voie de fait, l'administration peut être justiciable devant le juge judiciaire. Ce dernier a reçu, au Cameroun, le droit de connaître de certains actes de l'administration. Conformément à la loi, et au regard de la nouvelle tendance jurisprudentielle, le juge judiciaire a compétence pour apprécier la voie de fait (1.1); toutefois, cette prérogative est limitée en ce qu'elle est de nature à être paralysée par le soulèvement d'une exception préjudicielle (1.2).

\subsection{La compétence d'appréciation de la voie de fait administrative par le juge judiciaire}

L'appréciation de la voie de fait est une opération à double détente qui intègre à la fois la constatation, opération matérielle, et la qualification, opération intellectuelle.

\subsubsection{La constatation des agissements de l'administration par le juge judiciaire}

La constatation est un procédé par lequel un magistrat, dans la recherche de la vérité, observe et examine de manière concrète les éléments de fait nés des comportements ou des agissements d'une personne. Il s'agit d'un acte au moyen duquel le juge s'assure de l'existence des faits. Elle lui permet de voir concrètement comment les événements se sont produits ou de voir la manifestation de la vérité et d'apprécier rigoureusement l'exactitude des faits incriminés. Elle constitue une sorte d'enquête permettant au juge de trouver des indications et des éléments de nature à asseoir sa conviction ou de vérifier la sincérité de certaines allégations. Par la technique de la constatation, le juge procède à une sorte d'inspection judiciaire. Les éléments recueillis sont couchés sur un procès-verbal et servent de preuves les plus utiles pour la découverte de la vérité. La constatation judiciaire sert ainsi de base à un jugement ou à une décision de justice. C'est pourquoi elle commande la plus grande célérité puisqu'elle est déterminante pour la suite du procès. 
En droit camerounais, la constatation peut être faite soit à travers la mise en place d'une expertise ${ }^{13}$, soit à travers un transport judiciaire ordonné par le juge ${ }^{14}$. En matière de voie de fait, l'exploitation de la jurisprudence montre que le juge judiciaire fait généralement appel au transport judiciaire, c'est-à-dire à une descente sur les lieux afin d'apprécier la matérialité des faits. Dans l'affaire Tagatsing Tankou Fidèle contre communauté urbaine de Bafoussam, le juge des référés a ordonné «une descente immédiate sur les lieux à côté de la BICEC [...] à l'effet de constater ce qu'il appartiendra et pour d'éventuelles auditions ${ }^{15}$ ». Après ce transport judiciaire, le juge a déclaré ce qui suit: «Constatons qu'il y a voie de fait administrative de la Communauté Urbaine de Bafoussam au préjudice de TAGATSING TANKOU Fidèle à travers la fermeture abusive avec scellé de la boutique dénommée "la citadelle". Dans l'affaire Dongmo Étienne, Jiofack Jacques et Ngassa Édouard contre Monsieur le sous-préfet de l'Arrondissement de Nkong$\mathrm{Ni}$, le juge relève : «Attendu qu'une descente effectuée par nous, sur les lieux a permis de constater l'effectivité des scellés $n^{0} 19,20,21$ apposés en date du 2 décembre 2011 par Monsieur le sous-préfet de Nkong- $\mathrm{Ni}^{16}{ }$.

Aux termes de l'article 9 alinéa 4 de l'ordonnance $n^{0} 72 / 06 \mathrm{du}$ 26 août 1972 portant organisation de la Cour suprême, «les tribunaux de droit commun connaissent [...] en outre [...] des voies de fait administratives ${ }^{17} »$. Ces dispositions ont été reprises par le législateur de $2006^{18}$. En libellant ces dispositions comme telles, le législateur camerounais reconnaît clairement la compétence de constatation de la voie de fait administrative au juge judiciaire, puisque dans la logique, on ne peut pas connaître de la voie de fait sans l’avoir au préalable constaté.

13. Si, dans le contentieux administratif, l'expertise est prévue par les articles 84 à 93 de la loi 2006/022 du 29 décembre 2006 fixant l'organisation et le fonctionnement des tribunaux administratifs, devant le juge judiciaire, cette mesure d'instruction est prévue par les articles 116 à 128 du Code de procédure civile et commerciale.

14. Encore appelée, descente sur les lieux, cette mesure est prévue et régie par les articles 72 à 76 de la loi 2006/022 du 29 décembre 2006 fixant l'organisation et le fonctionnement des tribunaux administratifs et devant le juge judiciaire par les articles 113 à 115 du Code de procédure civile et commerciale.

15. Président du tribunal de première instance de Bafoussam, ord. $\mathrm{n}^{0} 119 / \mathrm{REF} / \mathrm{H}$ H/2016, 19 sept. 2016.

16. Président du tribunal de première instance de Dschang, ord. de référé $\mathrm{n}^{\circ}{ }_{21} / \mathrm{ORD}$, 8 déc. 2011.

17. Voir Ord. $\mathrm{n}^{0}$ 72/o6, 26 août 1972, art. 9, al. 4 portant organisation de la Cour suprême.

18. Voir L. $\mathrm{n}^{\mathrm{o}} 2006 / 022,29$ déc. 2006, art. 3 (2) fixant l'organisation et le fonctionnement des tribunaux administratifs. 
Ainsi, la législation camerounaise offre l'opportunité au juge judiciaire d'intervenir dans les différends mettant en cause l'administration. Cette situation est conforme aux exigences de l'administrative law, longtemps applicable dans le Cameroun anglophone. Selon la logique de cette branche du droit, les règles de droit privé dans leur forme et leur contenu, et suivant leurs procédures, doivent être appliquées par les juridictions de droit commun aux opérations dites administratives ${ }^{19}$.

Certes, depuis l'intervention de l'ordonnance du 26 août 1972 fixant l'organisation de la Cour suprême, le juge administratif a été considéré par la jurisprudence et par une partie majoritaire de la doctrine, comme étant la seule autorité compétente pour constater la voie de fait. À notre sens, telle n'est pas l'idée qui peut se dégager des dispositions législatives. À la lecture de l'article 9(4) de l'ordonnance de 1972/06 repris par l'article 3 (2) de la loi $\mathrm{n}^{0}$ 2006/022 fixant l'organisation et le fonctionnement des tribunaux administratifs, la compétence principale en matière de constatation de la voie de fait administrative revient au juge judiciaire. De manière claire, et selon l'idée qui peut aisément se dégager de l'article 9(4) de l'ordonnance de 1972 repris mutatis mutandis par l'article 3(2) de la loi de 2006, lorsqu'aucune partie au procès n'a contesté la compétence de constatation de la voie de fait administrative à travers l'invocation de l'exception préjudicielle, le juge judiciaire constate. On comprend dès lors à l'examen de l'article 3(2) de la loi $\mathrm{n}^{0}$ 2006/022 du 29 décembre 2006 que le législateur ne semble pas retirer cette compétence de constatation au juge judiciaire. Ce législateur a même tendance, en la matière, à faire de ce juge l'acteur principal. On pouvait tout de même opposer à cette situation juridique la violation du principe de la séparation des autorités administratives et judiciaires ${ }^{20}$. À notre sens, un tel argument serait impertinent en ce que dès lors qu'il y a voie de fait, il n'y a plus activité administrative et on peut logiquement en déduire une soumission complète de l'administration aux règles de droit privé ${ }^{21}$. Autrement dit, les voies de fait administratives sont

19. Éric Nfobin, «La résurgence de l'administrative law comme droit de l'administration au Cameroun anglophone: réflexion sur un phénomène peu connu ", Revue $E D J A, \mathrm{n}^{\mathrm{o}} 74,2007, \mathrm{p} .41$.

20. Sur ce principe, lire Jean-François Flauss, Les questions préjudicielles et le principe de la séparation des autorités administrative et judiciaire, thèse, Strasbourg, 1976, p. 267.

21. En effet, la voie de fait entraîne la volonté de sanctionner une attitude grossière de l'administration considérée comme particulièrement condamnable. La compétence administrative était un véritable privilège de l'administration. En cas de voie de fait, ce privilège devrait lui être retiré parce qu'elle a méconnu gravement le droit. 
des actes tellement grossiers et dénaturés qu'ils perdent leur caractère administratif. À cet effet, les actes pris ou posés par l'autorité ne peuvent plus être considérés comme rattachés à la compétence administrative. Ils sont dès lors dépouillés des avantages reconnus à l'administration. C'est parce que l'acte a perdu son caractère administratif que le juge judiciaire détient la plénitude de compétence (mais pas l'exclusivité) en matière de constatation de la voie de fait administrative.

À l'analyse de la jurisprudence camerounaise en matière de voie de fait, on s'aperçoit ainsi que la compétence de constatation est exercée courageusement et audacieusement par certains juges judiciaires ${ }^{22}$.

À côté de la compétence de constatation, s'est greffée la compétence de qualification qui est une suite logique de la constatation.

\subsubsection{La qualification des agissements de l'administration par le juge judiciaire}

La qualification est une opération intellectuelle qui consiste à déterminer, à la lumière des faits, la vraie nature juridique d'une opération matérielle. Elle consiste à classer certaines opérations matérielles dans une catégorie juridique, c'est-à-dire à leur trouver une nature juridique.

Le juge judiciaire ne peut donc pas connaître du contentieux de la voie de fait administrative sans passer par cette opération. Dans l'affaire opposant $\mathrm{M}^{\mathrm{me}}$ Pagui Tonfack Armelle au chef de centre départemental des impôts de la Menoua, receveur des impôts du centre départemental de la Menoua et Edjo'o Pierre Christian, le juge judiciaire après avoir constaté les agissements de l'autorité administrative les a qualifiés de voies de fait. In extenso, le juge judiciaire pense qu' «une telle décision, prise en marge des procédures, est manifestement illégale

22. À titre indicatif, on peut citer quelques cas: président du tribunal de première instance de Dschang, ord. $\mathrm{n}^{\mathrm{o}}$ 16/ORD, 8 mai 2014, $\mathrm{M}^{\mathrm{me}}$ Lontsi Hortense $\mathrm{c} / \mathrm{le}$ chef du centre des impôts de l'Ouest à Bafoussam et Mbiabot Lombo Moise; ord. de référé $\mathrm{n}^{0}$ 21/ORD, 8 déc. 2011, Kengmo Étienne et autres c/ M. le souspréfet de Nkong-Ni; ord. de référé $\mathrm{n}^{\mathrm{o}}$ 06/ORD, 15 déc. 2006, $\mathrm{M}^{\mathrm{me}}$ Pagui Tonfack Armelle c/ le chef du centre départemental des impôts de la Menoua et autres. De même, dans l'affaire Deido pharmacy, objet de l'ordonnance de référé $\mathrm{n}^{\circ} 791 / \mathrm{ORD}$, 10 avr. 2002 rendue par le président du tribunal de première instance de Douala, le juge a constaté la voie de fait administrative. Il en est de même de l'ordonnance $\mathrm{n}^{\mathrm{o}} 19 / \mathrm{ORD}, 16$ sept. 2008 rendue par le président du tribunal de première instance de Dschang dans l'affaire Succession Fokoua Pierre représentée par $\mathrm{M}^{\text {me }}$ Fokoua née Medie Augustine c/ la commune de Dschang et le $\mathrm{D}^{\mathrm{r}}$ Momo Bernard. Dans cette affaire, le président du tribunal de première instance de Dschang, statuant en matière de référé d'heure à heure, a constaté la voie de fait. 
et par conséquent constitutive de voie de fait ${ }^{23}$ ». Dans l'affaire qui oppose $\mathrm{M}^{\mathrm{me}}$ Lontsi Hortense au chef du centre des impôts de l'Ouest à Bafoussam, le juge déclare "qu'en apposant les scellés non pas sur les installations de la société DOTSADJI SARL, société débitrice, objet de la procédure de recouvrement forcé mais plutôt sur les portes de l'alimentation de l'espoir, les défendeurs ont commis une voie de fait». Dans l'affaire Succession Fokoua Pierre contre commune de Dschang et $\mathrm{D}^{\mathrm{r}}$ Momo Bernard, le juge de référé du tribunal de première instance de Dschang a jugé que les agissements du maire étaient constitutifs de « voie de fait ». De manière limpide, le juge affirme "qu'en terrassant ainsi ledit site et en autorisant les véhicules de transport public à s'y installer, le Maire commet une voie de fait puisqu'il prend une décision qui porte atteinte à une propriété privée et est insusceptible d'être rattachée à l'exécution d'un texte législatif ou réglementaire ${ }^{24} »$. Dans le cas Kengmo Étienne, Jiofack Jacques et Ngassa Édouard contre M. le sous-préfet de l'arrondissement de Nkong- $\mathrm{Ni}^{25}$, l'acte de saisine avait auparavant attiré l'attention du juge en des termes clairs, à savoir "qu'il y a là voie de fait justifiant l'intervention du juge judiciaire ». Rendant sa décision dans cette affaire, le juge estime qu' "en l'absence d'une procédure légale ou réglementaire, dans le cas d'espèce, les agissements du sous-préfet de Nkong-Ni participent d'une voie de fait ». En se comportant ainsi, le juge de l'ordre judiciaire pose de manière solide les bases d'une analyse originale en matière du contentieux de la voie de fait. Cette position rejoint la décision rendue par le président du tribunal de première instance de Douala dans l'affaire Deïdo Pharmacy contre le chef du centre divisionnaire des impôts de Douala VII ${ }^{26}$. Il en résulte ainsi que normalement l'acte devenu une simple voie de fait, et non plus une voie de droit, échappe à la compétence administrative et relève du juge judiciaire.

Mais, en la matière, le juge judiciaire n'est pas un automate. Après constatation de la situation, il peut estimer que les agissements de

23. Président du tribunal de première instance de Dschang, ord. de référé $\mathrm{n}^{\circ}$ 06/ORD, 15 déc. 2006.

24. Voir le président du tribunal de première instance de Dschang, ord. de référé $\mathrm{n}^{\mathrm{o}}$ 19/ORD, 16 oct. 2008.

25. Président du tribunal de première instance de Dschang, ord. de référé $\mathrm{n}^{\circ}{ }_{21 / O R D}$, 8 déc. 2011.

26. Président du tribunal de première instance de Douala, ord. de référé $n^{\circ} 791$, 10 janv. 2002. Dans cette affaire, le président du tribunal statuant en référé a constaté la voie de fait administrative et a ordonné la levée des scellés apposés sur l'immeuble exploité par la requérante, sous astreinte. 
l'administration soumis à son examen ne constituent pas une voie de fait. Il l'a réitéré en des termes clairs dans les affaires opposant la société de transport en commun à la communauté urbaine de Yaoundé ${ }^{27}$, l'agence de voyages Ndah dem à Ngnoufo Mama et la Délégation générale à la sûreté nationale ${ }^{28}$, Ngoumtsop Jeanne Salomène à la communauté urbaine de Bafoussam ${ }^{29}$. Face à cette situation où le juge estime qu'il n'y a pas voie de fait, il se déclare incompétent pour statuer sur la cause ou, à défaut, il déboute la partie demanderesse c'est-à-dire déclare son action non fondée.

Cette attitude s'inscrit dans une perspective d'évolution du droit. On peut aisément comprendre cette logique du droit découlant des textes camerounais. En fait, le législateur perçoit mal, à l'heure de la protection avérée des droits de l'homme et des libertés publiques, pourquoi un juge judiciaire, qui a le pouvoir de connaître de la voie de fait administrative, ne pourrait pas la qualifier, surtout si aucune partie n'a trouvé d'objection à travers le soulèvement d'une exception préjudicielle.

Mais, si une partie s'est opposée à l'exercice de cette compétence à travers le soulèvement in limine litis d'une exception préjudicielle, le juge se trouve coincé, car il est tenu de suspendre l'examen de la cause jusqu’à ce que l'exception préjudicielle soulevée soit définitivement tranchée. La compétence de constatation et de qualification reconnue au juge judiciaire a ainsi une portée limitée.

\subsection{La portée limitée de la compétence du juge judiciaire en matière d'appréciation de la voie de fait administrative}

$\mathrm{Au}$ cours du déroulement d'une procédure dans laquelle une voie de fait a été invoquée, une partie au procès, en l'occurrence la partie

27. Dans cette affaire, le juge déclare « qu'on ne saurait donc assimiler cet acte à une voie de fait, le délégué du gouvernement ayant agi en toute légalité; qu’il convient tout en recevant la Société de transport en commun en son action, de l'en débouter " (président du tribunal de première instance de Yaoundé, centre administratif, ord. $n^{\circ} 339 / \mathrm{C}, 9$ févr. 2006).

28. Dans cette espèce, le juge a estimé que les actes décriés de l'administration ne sont pas assimilés à des voies de fait administratives. Tribunal de première instance de Dschang, jugement $\mathrm{n}^{\circ} 21 / \mathrm{Civ}, 11$ sept. 2014.

29. Dans cette affaire, le juge affirme "qu'ainsi, la démolition de la barrière de la demanderesse procède de cette violation des règles d'urbanisme; qu'elle ne devrait donner lieu à aucune indemnisation, ni réparation ; qu'il échet de rejeter la demande de réparation de dame Ngountsop Jeanne Salomène comme non fondée » (Tribunal de première instance de Bafoussam, jugement $n^{\circ} 58 / \mathrm{Civ} / 2016,3$ juin 2016). 
défenderesse, peut soulever une exception préjudicielle aux fins de contestation de la qualification des faits (1.2.1). Face à cette situation, la loi impose au juge judiciaire, si l'exception est soulevée dans les formes et délais requis, un sursis à statuer jusqu'à l'intervention de la décision définitive qui sera rendue sur l'exception soulevée (1.2.2).

\subsubsection{Le soulèvement de l'exception préjudicielle}

Aux termes de l'article 3 (2) de la loi 2006/022 suscitée, «il est statué sur l'exception préjudicielle soulevée en matière de voie de fait administrative par la Chambre administrative de la Cour suprême ». On constate ainsi, en matière contentieuse, que l'idée de la question préjudicielle renvoie en principe, sauf dans certains cas, à l'incompétence du juge saisi au préalable et devant qui la question est soulevée ${ }^{30}$. Le professeur Gérard Cornu analyse la question préjudicielle comme «le point litigieux dont la solution doit précéder celle de la question principale qu'elle commande mais qui ne peut être tranchée par la juridiction saisie, de telle sorte que celle-ci doit surseoir à statuer jusqu'à ce que la question préjudicielle ait été résolue par la juridiction seule compétente pour en connaître ${ }^{31} »$. La question préjudicielle est donc celle qui appelle un jugement distinct et séparé émanant d'un juge autre que celui du fond et dont la solution doit précéder l'examen du fond et être tranchée par une juridiction autre que celle compétente sur l'action principale. En d'autres termes, la question est qualifiée de préjudicielle chaque fois que le règlement relève d'une autre juridiction que celle devant laquelle ladite question est soulevée ${ }^{32}$. C'est donc parce que le juge préalablement saisi est incompétent pour répondre à la question

30. D’après le Tribunal de conflit français, lorsqu'une question préjudicielle est identique à une question sur laquelle la cour de justice a déjà été amenée à se prononcer ou lorsque la réponse à cette question ne laisse place à aucun doute raisonnable ou peut être clairement déduite de la jurisprudence, la cour peut, après avoir entendu l'avocat général, statuer par voie d'ordonnance motivée, en faisant notamment référence à l'arrêt déjà rendu sur cette question ou à la jurisprudence pertinente (T. confl., 12 déc. 2011, Société Green Yellow et autres c/ Électricité de France, $\mathrm{n}^{\mathrm{o}} 3841$ ).

31. Gérard Cornu (dir.), Vocabulaire juridique, Paris, PUF, 2001, p. 703.

32. Édouard Laferrière, Traité de juridictions administratives, Paris, Berger-Levrault, t. I, 1896, p. 444 . 
qu'elle est dite préjudicielle ${ }^{33}$. Sans la réponse à la question posée au juge de renvoi, le juge saisi au principal est impuissant à résoudre le litige qui lui est soumis ${ }^{34}$. En matière de voie de fait, le législateur de 2006, reprenant son devancier de 1972, affirme qu' "il est statué par la Chambre administrative de la Cour suprême sur l'exception préjudicielle soulevée en matière de voie de fait administrative... ». Certes, ces dispositions sont imprécises, car elles ne déterminent pas qui peut soulever l'exception, à quel moment de la procédure cette exception peut être soulevée et sur quoi elle va porter, autrement dit, cette exception porte-t-elle sur la constatation ou la qualification, sur la réparation ou sur la cessation de la voie de fait?

Face à cette imprécision, la jurisprudence et la doctrine se sont efforcées d'apporter quelques clarifications. L'exception préjudicielle peut être en principe soulevée par la partie au procès ayant intérêt; mais elle pourrait aussi l'être à titre exceptionnel par un juge qui se trouve embarrassé. Dans cette dernière hypothèse, on parle de renvoi préjudiciel. Cette exception ne porte pas sur la réparation ou la cessation de l'action, mais porte exclusivement sur la constatation ou la qualification. N'étant pas d'ordre public, elle doit, pour avoir de chances de prospérer, être soulevée en principe avant toutes conclusions au fond. Aux termes du paragraphe premier de l'article $97 \mathrm{du}$ Code de procédure civile et commerciale, «toutes les exceptions, demandes en nullité, fins de non-recevoir et tous les déclinatoires visés aux articles précédents sauf l'exception d'incompétence rationae materiae et l'exception de communication de pièces, seront déclarés non recevables s'ils sont présentés après qu'il aura été conclu au fond». Le juge l'a réitéré dans l'affaire Société Bansoa Airlines SARL (Grand Ouest) contre CNPS, Ebongo Samuel, centre départemental des impôts de Dschang et Bounnang P. Désiré ${ }^{35}$. De plus, cette exception doit être présentée simultanément aux autres, au risque d'être frappée d'irrecevabilité. Pour

33. Lire à ce sujet, Claudie Boiteau, «Questions préjudicielles devant le juge administratif», JCl. justice administrative, Fasc. 36, p. 3 et suiv.; Axel Wandji Kemajou, La question préjudicielle dans le contentieux administratif camerounais, mémoire de master en droit public, Université de Dschang, 2012, $140 \mathrm{p}$.

34. Daniel Granjon, «Les questions préjudicielles », RFDA 1968, p. 78 et suiv.

35. Président du tribunal de première instance de Dschang, ord. $n^{\circ}$ o7/ORD, 12 juin 2008. Le juge de manière claire déclare "qu'en l'espèce, il est évident que la juridiction de céans s'était déjà prononcée sur l'exception d'incompétence préalablement soulevée par les défendeurs; que celle relative au sursis à statuer devient dès lors tardive; que bien plus les défenseurs qui sollicitent le sursis à statuer ont conclu au fond; qu'il échet dès lors de rejeter cette demande comme tardive». 
le législateur, "toutes les autres exceptions, demandes de nullité, fins de non-recevoir et tous les autres déclinatoires doivent être proposés simultanément et aucun ne sera plus reçu après un jugement statuant sur l'un d'eux ${ }^{36}{ }$. En cas d'invocation d'une exception préjudicielle ou en cas d'un renvoi préjudiciel dans les formes légales, la constatation ou la qualification en matière de voie de fait relève de la compétence exclusive de la chambre administrative de la Cour suprême ${ }^{37}$.

Le législateur camerounais accorde ainsi un privilège à la juridiction administrative pour constater et qualifier la voie de fait en cas d'invocation de l'exception préjudicielle devant le juge judiciaire. Il lui revient donc de pouvoir apprécier les actes de l'administration. Ce qui permet d'éviter la confusion entre voie de fait administrative et notions voisines à savoir emprise irrégulière, excès de pouvoir, circonstances d'exception, référé-liberté ${ }^{38}$. C'est donc l'évocation de la question préjudicielle en principe par l'une des parties au procès ayant intérêt qui est de nature à mettre fin à la compétence de constatation et de qualification du juge judiciaire dans le litige en question. Ainsi, l'invocation de la question préjudicielle, lorsqu'elle répond aux exigences légales, enlève la compétence de constatation ou de qualification au juge judiciaire. À la vérité, l'invocation de cette question dès cet instant le rend ipso facto, si elle est fondée, incompétent pour constater et qualifier la voie de fait ${ }^{39}$. Y faisant suite, le juge est tenu, à moins de violer la loi, d'ordonner un sursis

36. Code de procédure civile et commerciale, art. 97, dernier paragraphe.

37. L. $\mathrm{n}^{\mathrm{0}} 2006 / 022,29$ déc. 2006, art. 3 (2), fixant l'organisation et le fonctionnement des tribunaux administratifs et $\mathrm{L} . \mathrm{n}^{\circ}$ 2006/016, 29 déc. 2006, 38, al. (c) fixant l'organisation et le fonctionnement de la Cour suprême.

38. Voir sur cette question, Bonaventure Carol Mballa Emotho, Voie de fait administrative et compétences juridictionnelles au Cameroun, mémoire de master en droit public, Université de Dschang, 2008-2009, p. 10-18.

39. Pour qu'une question préjudicielle soit admise par le tribunal principalement saisi, il faut, d'une part, qu'elle soulève une difficulté sérieuse, c'est-à-dire une difficulté « de nature à faire naître un doute dans un esprit éclairé» (Édouard Laferrière, Traité de la juridiction administrative, cité par André de Laubadère, note sous CE, ass., 19 juin 1964, Société des pétroles Shell-Berre et autres, AJDA, 1964, II, p. 443), d'autre part, que la solution du juge de renvoi soit nécessaire au jugement du fond, c'est-à-dire que le sort du litige en dépende. De façon générale, le mécanisme de la question préjudicielle n'est mis en œuvre que sous la double réserve de l'utilité de la question posée pour la solution du litige et de son caractère sérieux (Yves Gaudemet, "Les questions préjudicielles devant les deux ordres de juridiction ", RFDA 1990, p. 769 . 
à statuer dans la cause ${ }^{40}$ jusqu'à ce que soit tranchée définitivement la question de droit relevant de la compétence d'une juridiction de l'autre ordre ${ }^{41}$. On peut tout de même relever que, dans de nombreux cas, cette exception préjudicielle est difficilement soulevée lors des débats. Elle est très souvent mise aux oubliettes, peut-être à cause de l'ignorance des justiciables.

Mais, en attendant que cette exception soit soulevée, le juge judiciaire détient la pleine compétence en cette matière. C'est dire que si, lors de la qualification des faits qui figurent généralement dans l'acte de saisine du tribunal, aucune partie ne soulève de contestations à travers une exception préjudicielle, le juge judiciaire constate et précise, au regard des éléments de fait et de droit dont il dispose, la véritable nature juridique des agissements de l'administration.

$\mathrm{Si}$, par extraordinaire, une partie au procès, et plus précisément le défendeur, soulève cette exception préjudicielle avant toutes conclusions au fond, le juge judiciaire doit s'abstenir de constater ou de qualifier. Il est tenu, au regard de la situation, d'ordonner en principe un sursis à statuer $^{42}$.

\subsubsection{Le sursis à statuer}

Le sursis à statuer est la décision d'une juridiction de reporter le cours d'un jugement, dans l'attente de la production d'un événement. Principe général de la procédure, le sursis à statuer est une décision du juge opérant suspension provisoire du cours de l'instance jusqu'à l'accomplissement d'une formalité ou jusqu'à ce que soit rendue la décision d'une autre juridiction devant laquelle se trouve engagé un autre procès qui n'est pas encore jugé et qui aura une influence sur le sort de la cause dont le tribunal est actuellement saisi. Ainsi, sur décision du juge, l'instance est suspendue provisoirement par un obstacle qui, tant qu'il n’est pas résolu, empêche de parvenir à une décision au fond. Pour que

40. Il importe de souligner que le sursis à statuer permet d'éviter une contradiction avec la solution qu'une juridiction administrative pourrait retenir si elle était saisie des mêmes faits.

41. Lire Clovis Callet, «La fonction juridictionnelle à l'épreuve de la question préjudicielle. Regard sur les fonctions de la question préjudicielle», Jurisdoctoria, $\mathrm{n}^{\circ} 6$, 2011, p. 17 et suiv.

42. Le tribunal de grande instance du Mfoundi l'a réitéré dans l'affaire Kitimbi Pierre c/ communauté urbaine de Yaoundé, car, par jugement avant dire droit $\mathrm{n}^{\circ} 220 \mathrm{du}$ 22 avril 1987, il a ordonné un sursis à statuer et a renvoyé la partie la plus diligente à saisir le juge administratif aux fins de constatation et de qualification de la voie de fait. 
le sursis à statuer soit prononcé en droit camerounais, en matière de voie de fait, l'exception préjudicielle soulevée doit être déclarée fondée. Or, pour être fondée, l'exception doit être soulevée avant toutes conclusions au fond par la partie ayant intérêt ${ }^{43}$. C'est dire que le juge n'est pas tenu de prononcer le sursis à statuer lorsqu'il est demandé par une partie au procès. Le juge l'a réitéré en rejetant une demande de sursis à statuer en matière de voie de fait dans l'espèce opposant les ayants droit de feu Tchatchou à la communauté urbaine de Yaoundé, au motif de ce que «le débat sur l'objet et le bien-fondé de la demande était déjà engagé à travers la réclamation de l'expertise " présentée par la défenderesse ${ }^{44}$.

En principe, pour que le sursis soit prononcé, il faut que la question préjudicielle soulevée soit pertinente, raisonnable et apte à contribuer à la bonne administration de la justice. Certes, en matière de voie de fait, le juge est lié par l'invocation de l'exception préjudicielle lorsqu'elle est faite avant toutes conclusions au fond et de manière simultanée avec les autres exceptions. C'est pourquoi, au Cameroun, l'exception préjudicielle en matière de voie de fait a un caractère spécifique. La spécificité vient de ce que lorsque l'exception en matière de voie de fait a été soulevée avant toutes conclusions au fond et, si besoin est, de manière simultanée avec les autres exceptions, demandes de nullité, fins de non-recevoir et tous les autres déclinatoires, du moins, avant que le jugement sur l'un d'eux ne soit rendu, le juge n'a plus le droit d'apprécier sa pertinence ou son bien-fondé. Il est tenu d'ordonner le sursis à statuer même s'il est convaincu que la démarche du demandeur à l'exception vise à prolonger inutilement la procédure, c'est-à-dire, frise le dilatoire. On comprend dès lors qu'en matière de voie de fait, le sursis à statuer est le plus souvent imposé par le législateur, de sorte que le juge se trouve obligé de le prononcer lorsqu'il est demandé in limine litis et, si besoin est, de manière simultanée avec les autres exceptions, demandes de nullité, fins de non-recevoir et tous les autres déclinatoires.

Il importe de rappeler que le sursis à statuer ne dessaisit pas la juridiction qui sursoit à statuer. L'instance devant cette juridiction est simplement suspendue ${ }^{45}$. Une fois qu'il a été statué sur la question ayant entraîné le sursis, la procédure suspendue peut être reprise sans

43. Code de procédure civile et commerciale, art. 97.

44. Tribunal de première instance de Yaoundé, centre administratif, ord. $n^{\circ} 335 / C$, 30 juin 2009. Il s'agit d'une décision du juge judiciaire statuant en matière de référé.

45. Robert Assontsa, note sous arrêt $\mathrm{n}^{\circ}$ 134/C, 20 juil. 2012, affaire Communauté urbaine de Douala c/ famille Yamilo représentée par son chef Ebongue Doumbe Albert, Juridis Périodique, ${ }^{\circ}$ 102, 2015, p. 66. 
formalités particulières. Le sursis à statuer est donc la conséquence directe qui découle du soulèvement de l'exception préjudicielle. Il peut être d'office ordonné par le juge, ceci lorsqu'il estime souverainement, c'est-à-dire de son propre chef, que l'événement dont il attend la réalisation est nécessaire à l'appréciation du différend pour lequel il est saisi. Il peut aussi être ordonné à la demande des parties au procès. Face à cette situation, le juge, dans sa décision de sursis, renvoie la partie la plus diligente à mieux se pourvoir notamment à saisir le juge administratif compétent aux fins de qualification ${ }^{46}$. C'est d'ailleurs ce qu'il s'est passé dans les espèces Kitimbi Pierre contre communauté urbaine de Yaoundé, Ena Mballa Hubert contre la même structure, $\mathrm{M}^{\mathrm{me}}$ Balanos née Karminos Chloé contre commune rurale d'Obala, $\mathrm{M}^{\text {mes }}$ Mbarga Julienne et Ngono Mimbang contre communauté urbaine de Yaoundé ${ }^{47}$.

Deux cas de figure peuvent se présenter à cette étape: si le juge administratif estime qu'il n'y a pas voie de fait, le juge judiciaire, à moins d'être en présence d'un fait administratif, se déclare incompétent et renvoie la partie la plus diligente à se mieux pourvoir. À ce niveau, si l'on est en présence d'un simple acte administratif illégal, la victime ne pourrait que saisir le juge administratif qui statuera de nouveau en contentieux d'excès de pouvoir ou en indemnisation ${ }^{48}$. Dans un pareil cas, la victime, conformément au jugement rendu dans l'affaire $D^{r}$ Ndzietam Pierre contre État du Cameroun ${ }^{49}$, peut saisir directement le juge par un recours contentieux sans passer préalablement par un recours gracieux. $\mathrm{Si}$, en revanche, le juge administratif estime qu'il y a voie de fait administrative, l'affaire est retournée au juge judiciaire

46. Si, hier, en cas de soulèvement d'une exception préjudicielle en matière de voie de fait, le juge administratif compétent pour qualifier, sur la base de l'ordonnance $\mathrm{n}^{\mathrm{o}} 72 / 06$ du 26 août 1972, était l'assemblée plénière de la Cour suprême, aujourd'hui, à la faveur de la loi $\mathrm{n}^{\mathrm{O}}$ 2006/022 du 29 décembre 2006, c'est la chambre administrative de la Cour Suprême qui est désormais compétente, voir art. 3 (2).

47. CS/AP, arrêt $n^{\circ}$ 18/A, 27 mai 2010; CS/CA, jugement $n^{\circ}$ 39/92-93, 29 avr. 1993; CS/AP, arrêt n ${ }^{\circ}$ 68/A, 22 févr. 2007, CS/AP, arrêt n ${ }^{\circ}$ 14/A, 27 mai 2010.

48. C'est ce qui s'est passé dans l'espèce Ministère public c/ Fotsa Patrice Désiré, État du Cameroun, CS/AP, arrêt n ${ }^{\circ}$ 102/A, 13 déc. 2007. Dans cette affaire, l'assemblée plénière de la Cour suprême a estimé que les agissements de l'État du Cameroun ne constituaient pas une voie de fait administrative, mais un simple acte administratif justiciable devant le juge administratif.

49. CS/CA, jugement $n^{\circ} 47,25$ mai 1982. 
pour cessation et réparation ${ }^{50}$. On comprend dès lors que la requête traduisant l'administration devant le juge judiciaire peut aboutir à la condamnation de celle-ci.

\section{La condamnation de l'administration devant le juge judiciaire}

L'administration traduite devant le juge judiciaire peut faire l'objet de condamnation si les éléments à conviction sont réunis. Sous cet angle, elle peut être condamnée à la cessation de la voie de fait (2.1) et/ou à la réparation du préjudice causé par ses agissements (2.2).

\subsection{La condamnation à la cessation des agissements constitutifs de voie de fait}

Le fondement juridique de la compétence du juge judiciaire pour ordonner toute mesure visant à mettre fin à la voie de fait administrative est connu en droit camerounais (2.1.1). Y faisant suite, le juge, en se référant à ce fondement, prend régulièrement des mesures visant à faire cesser ces actes dénaturés de l'administration (2.1.2).

\subsubsection{Le fondement de la compétence du juge judiciaire en matière de cessation de la voie de fait administrative}

Lorsque l'administration commet une illégalité grossière qui menace gravement la liberté ou la propriété d'un individu, on considère que ses actes perdent leur caractère administratif et que, dès lors, elle a commis une voie de fait ${ }^{51}$. Le juge judiciaire étant le juge de droit commun de protection de la liberté individuelle et de la propriété, il n'est que logique que ce soit lui qui soit compétent en la matière pour, après constatation et qualification, ordonner toutes mesures pour y mettre un terme.

$\mathrm{Au}$ Cameroun, une telle analyse est admise tant par la jurisprudence que par les textes qui l'ont entérinée.

50. Les affaires Ena Mballa Hubert et Kitimbi Pierre c/ communauté urbaine de Yaoundé sus-évoquées illustrent parfaitement cette hypothèse. Il en est de même de l'affaire de $\mathrm{M}^{\mathrm{me}}$ Balanos née Karminos Chloé c/ la commune rurale d'Obala (CS/AP, arrêt $n^{\circ}$ 62/A/CS/AP, 22 févr. 2007) où le juge administratif a estimé qu'il y avait voie de fait et a renvoyé les parties devant le juge judiciaire pour réparation (lire à ce sujet la note de Lionel Pierre Guessele Isseme, RADP 2012, p. 17-28).

51. Voir Mohammed Amine Benabdallah, «La voie de fait administrative en droit marocain ", Mélanges offerts au doyen Yadh Ben AChour, Tunis, Centre de publication universitaire, 2008, p. 9 et suiv. 
Après l'arrêt Mve Ndongo Abraham, une série d'affaires est venue illustrer la compétence du juge judiciaire en matière de prescription de toute mesure pour mettre fin à la voie de fait ${ }^{52}$. Entérinant la jurisprudence, le législateur a donné une assise textuelle à cette compétence. Aux termes de l'article 9 alinéa 4 de l'ordonnance $n^{0} 72 / 06$ du 26 août 1972 portant organisation de la Cour suprême, les tribunaux de droit commun « connaissent, en outre, [...] des voies de fait administratives et ordonnent toute mesure pour qu'il y soit mis fin ». Ces dispositions sont reprises par la loi $\mathrm{n}^{\mathrm{O}}$ 2006/022 du 29 décembre 2006 fixant l'organisation et le fonctionnement des tribunaux administratifs ${ }^{53}$.

C'est donc en conformité avec le droit positif que le juge dans les affaires Pagui, Tagatsing Tankou Fidèle, $\mathrm{M}^{\mathrm{me}}$ Lontsi Hortense et autres s'est déclaré compétent dans l'affaire à lui soumise et pour ordonner toute mesure mettant fin à la voie de fait constatée. On peut remarquer que le juge évoque tantôt l'article 9 alinéa 4 de l'ordonnance $n^{0} 72 / 6 \mathrm{du}$ 26 août 1972, tantôt, et ce depuis le 29 décembre 2006, l'article 3 alinéa 2 de la loi 2006/022 du 29 décembre 2006 comme soupape juridique de sa compétence. En se déclarant compétent pour juger l’administration face aux actes dénaturés ${ }^{54}$, le juge judiciaire confirme par là son rôle de gardien naturel des droits et libertés individuels ${ }^{55}$.

Il faut toutefois relever que l'atteinte à une liberté ou à un droit ne suffit pas pour que puisse être utilement invoquée la commission d'une voie de fait administrative. Il faut encore que cette atteinte soit particulièrement grave ${ }^{56}$. Comme le soulignait un auteur, la gravité du vice ou du manquement juridique doit rendre la mesure manifestement insusceptible de se rattacher à un pouvoir conféré par l'administration pour assurer le bon fonctionnement d'un service public ${ }^{57}$. L'atteinte peut

52. CS/CA, jugement $n^{0}$ 12/81-82, 28 janv. 1982, $M^{\text {me }}$ Binam née Ngo Njom Fidèle c/ État du Cameroun; CS/CA, jugement $n^{\circ}$ 39/92-93, 29 avr. 1993, ENA Mballa Hubert $\mathrm{c} /$ communauté urbaine de Yaoundé; CS/AP, arrêt $\mathrm{n}^{\circ}$ 20/A, 4 janv. 2001, Djotoum Henriette cl État du Cameroun (MINAT); CS/AP, arrêt $n^{\circ} 41 / \mathrm{A}$, 5 avr. 2001, Samedjeu Jean-Jacques c/ État du Cameroun; etc.

53. Voir art. 3, al. 2.

54. Lire Pierre Claude Awouafack, Le juge judiciaire face aux actes administratifs dénaturés: cas du tribunal de Dschang de 2006-2016, mémoire de master de droit public, Université de Dschang, 2016, p. 23-63.

55. Philippe Breton, L'autorité judiciaire gardienne des libertés essentielles et de la propriété privée, Paris, LGDJ, 1964, p. 19 et suiv.

56. Tribunal de grande instance du Mfoundi, jugement civil $\mathrm{n}^{\circ} 97,4$ janv. 1984, $\mathrm{M}^{\mathrm{me}}$ Etong $\mathrm{c} /$ Commune de plein exercice de Yaoundé; jugement civil $\mathrm{n}^{\mathrm{o}} 703$, 22 août 1990, Nie Foko Pascal c/ Commune urbaine de Yaoundé.

57. Voir Maurice Kamto, «La mise en cause des autorités administratives... », art. cité, p. 346-347. 
s'exprimer par les modalités d'exécution d'une décision, et au-delà par les libertés qu'ont pu prendre des agents semblant être dans l'ignorance des garanties que l'administration doit accorder aux administrés afin que leurs droits et libertés soient préservés. Dans l'espèce Pagui Tonfack, par exemple, les autorités ainsi que les agents du fisc de la localité ont ignoré les bornes de leurs pouvoirs et les textes qui définissent leurs compétences. Dans une telle hypothèse, souligne la doctrine, «ce n'est plus l'administration qui agit, c'est, à la limite, une force qui s'est échappée de l'État de droit ${ }^{58}{ }$. Cette situation de fait, vu l'urgence, nécessite généralement l'intervention du juge des référés, qui n'a malheureusement pas pouvoir pour réparer le préjudice (de peur de préjudicier le fond), mais qui détient pleine compétence pour faire cesser les agissements de l'administration, c'est-à-dire mettre un terme à la voie de fait entreprise ${ }^{59}$.

\subsubsection{L'adoption par le juge judiciaire des mesures nécessaires pour faire cesser la voie de fait}

Le juge judiciaire a compétence pour mettre fin à la voie de fait ${ }^{60}$, car le caractère grave des agissements déchoit l'administration du privilège de juridiction et par conséquent de l'application du droit administratif qui confère à cette administration le bénéfice des règles exorbitantes du droit commun ${ }^{61}$. Il s'agit de remettre les choses en l'état en ordonnant toutes mesures visant à mettre fin à la voie de fait. Ces mesures peuvent prendre la forme de restitutions ${ }^{62}$,

58. Jean-François Lachaume et Hélène Pauliat, Droit administratif, Paris, PUF, 2007, p. 347 .

59. Lire à ce sujet, Hassan Ouazzani Chahdi, «La voie de fait administrative et la compétence du juge des référés ", $R M D, \mathrm{n}^{\circ} 3,1985$, p. 157.

60. Dans l'affaire $\mathrm{M}^{\mathrm{me}}$ Pagui Tonfack Armelle ci-dessus évoquée, le juge judiciaire a réitéré cette compétence consacrée par la loi. Pour lui, «une telle décision, prise en marge des procédures, est manifestement illégale et par conséquent constitutive de voie de fait». "Que le juge judiciaire est compétent pour y mettre fin. »

61. En effet, lorsque l'administration a pris « un acte manifestement insusceptible de se rattacher d'une manière quelconque à l'exercice des pouvoirs dont elle est investie ", elle perd le bénéfice de l'application de la matière administrative pour devenir justiciable des règles du droit civil.

62. T. confl., 17 mars 1949, Société immobilière Rivoli-Sépastopol. 
d'enlèvement des scellés irrégulièrement apposés ${ }^{63}$, de destructions ${ }^{64}$, d'expulsions ${ }^{65}$, de la libération des personnes détenues ${ }^{66}$, de la levée d'une suspension ou d'une fermeture ${ }^{67}$, de l'enlèvement de matériels déposés sur un terrain privé ${ }^{68}$, de l'arrêt des travaux projetés ou en cours $^{69}$, etc. Ainsi, dans l'affaire Pagui Tonfack Armelle, le juge a ordonné «l'enlèvement des scellés apposés sur les magasins et stands de la demanderesse » et a commis "pour y procéder tel huissier territorialement compétent ». Dans l'affaire Dongmo née Tsague Marceline contre le receveur principal des impôts de l'Ouest à Bafoussam et autres ${ }^{70}$, le juge a déclaré «qu'il s'ensuit que les scellés apposés en date du 30 mai 2008 sur le magasin "la vie du paysan" l'ont été abusivement et encourent enlèvement ». Dans l'affaire Dongmo Étienne, Jiofack Jacques et Ngassa Édouard contre M. le sous-préfet de l'arrondissement de Nkong-Ni, le juge judiciaire a ordonné l'enlèvement des scellés $n^{0} 19,20,21$ apposés en date du 2 décembre 2011 par le sous-préfet de Nkong-Ni. Il a par la suite commis tout huissier territorialement compétent pour y procéder. Il en est de même dans l'affaire Lontsi Hortense contre le chef de centre

63. Président du tribunal de première instance de Dschang, ord. de référé $\mathrm{n}^{\circ}{ }_{21} / \mathrm{ORD}$, 8 déc. 2011, ord. $\mathrm{n}^{\mathrm{o}}$ 17/ORD, 14 août 2008, $\mathrm{M}^{\mathrm{me}}$ Dongmo née Tsague Marceline contre le receveur principal des impôts de l'Ouest à Bafoussam, Fogang Jean et autres.

64. Ces destructions porteront sur les constructions illégalement faites par l'administration sur un terrain appartenant à un particulier. Il s'agit des constructions effectuées en dehors de toute procédure d'expropriation.

65. T. confl., 17 mars 1949, Hôtel du Vieux Beffroi; CS/CA, jugement $n^{0} 31 / A D D /$ 04-05, 29 déc. 2004, Yikam Jérémie c/ État du Cameroun (MINDEF); président du tribunal de première instance de Dschang, ord. de référé $\mathrm{n}^{\circ}$ 19/ORD, 16 sept. 2008, Succession Fokoua Pierre représentée par $\mathrm{M}^{\mathrm{me}}$ Fokoua née Medie Augustine c/ la commune de Dschang et le $\mathrm{D}^{\mathrm{r}}$ Momo Bernard.

66. Affaire Retired justice Nyo'Wakai and 172 others vs the people, suit $\mathrm{n}^{\circ} \mathrm{C} / 19$ / CMR / 921, 23 déc. 1992. Dans cette espèce, le juge a ordonné la libération des personnes irrégulièrement détenues à la suite de contestations des résultats de l'élection présidentielle de 1992.

67. Président du tribunal de première instance de Douala, ord. de référé $\mathrm{n}^{\circ}$ 791/ORD, 10 avr. 2002, Deido pharmacy contre le chef du centre divisionnaire des impôts de Douala VII.

68. T. confl., 30 juin 1969, Société civile immobilière des Praillons.

69. Président du tribunal de première instance de Yaoundé, centre administratif, ord. de référé $n^{\circ} 335 / C$, 30 juin 2009, ayants droit de feu Tchatchou c/ communauté urbaine de Yaoundé.

70. Président du tribunal de première instance de Dschang, ord. $\mathrm{n}^{\circ}$ 07/ORD, 12 juin 2008, Société Bansoa-Airlines SARL (Grand Ouest) c/ CNPS, Ebongo Samuel, centre départemental des impôts de la Menoua et Bounnang P. Désiré; ord. $\mathrm{n}^{\mathrm{o}}{ }_{17} / \mathrm{ORD}, 14$ août $2008, \mathrm{M}^{\mathrm{me}}$ Dongmo née Tsague Marceline contre le receveur principal des impôts de l'Ouest à Bafoussam, Fogang Jean et autres. 
des impôts de l'Ouest à Bafoussam et Mbiabot Lombo Moïse. Le juge judiciaire dans cette affaire a ordonné l'enlèvement des scellés apposés le 28 avril 2014 par M. Mbiabot Lombo Moïse, agissant au nom du chef de centre principal des impôts de l'Ouest sur les portes de «l'alimentation de l'espoir». Il a, en cette même circonstance, commis tout huissier de justice territorialement compétent pour le faire. Le président du tribunal de première instance de Bafoussam épouse cette démarche puisque, dans l'affaire Tagatsing Tankou Fidèle contre la communauté urbaine de Bafoussam, il a ordonné «la levée immédiate par la Communauté Urbaine de Bafoussam par son service d'hygiène du scellé apposé sur les portes de ladite boutique et partant son ouverture forcée ce, sous astreinte de 25000 francs par jour de retard à compter de la date de signification de la présente ordonnance ${ }^{71} "$.

Comme on peut le constater, les mesures sus-évoquées peuvent être accompagnées d'astreinte ${ }^{72}$. Le juge peut aussi, au regard de l'urgence, assortir son jugement de l'exécution sur minute et avant enregistrement. Sur ce point, l'huissier ne pourrait être empêché dans son action d'exécution de cette décision de justice qu'en présence d'une défense à exécution délivrée en bonne et due forme par le greffier en chef de la juridiction supérieure à celle qui a rendu la décision et régulièrement notifiée à la partie exécutante. Ces solutions sont heureuses sur le plan pratique, car elles assurent efficacement la défense des administrés contre les atteintes portées aux droits et libertés par l'administration. Dans tous les cas, le juge judiciaire est doté d'une compétence lui permettant de prendre toute mesure pouvant mettre fin à la voie de fait administrative. Il le fait en vertu de ses pouvoirs d'injonction et d'astreinte ${ }^{73}$. On comprend dès lors que lorsque l'administration sort du

71. Président du tribunal de première instance de Bafoussam, ord. $\mathrm{n}^{0} 119 / \mathrm{REF} /$ H-H/2016, 19 sept. 2016; ord. $n^{\circ}$ 16/ORD, 8 mai 2014.

72. Dans l'affaire Deido pharmacy, objet de l'ordonnance de référé $n^{\circ} 791 / O R D d u$ 10 avril 2002 rendue par le président du tribunal de première instance de Douala, le juge a ordonné la levée des scellés apposés sur l'immeuble exploité par la requérante sous astreinte d'un montant à payer par jour de retard. Il en est de même de l'ordonnance $\mathrm{n}^{\circ} 19$ /ORD du 16 septembre 2008. Dans cette affaire, le président du tribunal de première instance de Dschang, statuant en matière de référé d'heure à heure, a constaté la voie de fait et a ordonné l'expulsion des défendeurs de l'immeuble occupé sous astreinte de 20 ooo $\mathrm{F}$ par jour de retard à compter du prononcé de l'ordonnance. Dans l'affaire Tagatsing Tankou Fidèle c/ communauté urbaine de Bafoussam suscitée, le juge judiciaire a ordonné la levée immédiate du scellé par la communauté urbaine sous astreinte de $25000 \mathrm{~F}$ par jour de retard à compter de la date de signification de l'ordonnance rendue.

73. Voir René Chapus, Droit administratif général, Paris, Montchrestien, t. 1, 1993, p. 690. 
cadre dans lequel doit s'inscrire son action, c'est la juridiction ordinaire, gardienne des droits et libertés, qui est compétente pour mettre fin à la voie de fait $^{74}$. Si les agissements de l'administration ont causé un préjudice à l'administré, le juge judiciaire en matière de voie de fait a la compétence exclusive pour ordonner la réparation.

\subsection{La condamnation à la réparation du préjudice né de la voie de fait administrative}

En cas de voie de fait, l'administration peut être condamnée par le juge judiciaire à réparer le préjudice subi. Il ne pourrait en être autrement, car «tout fait quelconque de l'homme qui cause un préjudice à autrui oblige celui par la faute duquel il est arrivé à le réparer ${ }^{75} »$. En droit camerounais, seul le juge du fond a compétence pour ordonner la réparation. En la matière, le juge de référé est incompétent. En matière de réparation du préjudice, le principe est celui de la réparation intégrale, sans perte ni profit pour la victime. Le principe de la réparation intégrale est celui en vertu duquel le responsable du préjudice doit indemniser tout le dommage et uniquement le dommage sans qu'il en résulte ni appauvrissement ni enrichissement de la victime. Ce préjudice, objet de la condamnation, peut être matériel ou moral.

\subsubsection{La condamnation à la réparation du préjudice matériel}

Le préjudice matériel est celui qui concerne les biens et actifs de la victime. Il constitue une atteinte à son patrimoine. Ce préjudice est la conséquence du dommage subi par la victime d'un acte ou d'un fait juridique dû à une atteinte aux biens immédiatement susceptibles d'évaluation pécuniaire et des préjudices corporels qui englobent quant à eux toutes les conséquences d'une lésion du corps humain, qu'elles soient d'ordre patrimonial ou non patrimonial. Le préjudice matériel peut émaner d'une atteinte aux biens meubles ou immeubles de la victime, une perte ou une privation de sa rémunération. Plus généralement, on admet que le préjudice matériel englobe le gain manqué et la perte éprouvée par la victime, économiquement, du fait de la faute.

Pour faire valoir droit à réparation du préjudice matériel en tant que victime, il faut démontrer préalablement une faute, un lien de causalité

74. Voir Philippe Breton, op. cit., p. 20 et suiv; Maurice Kamto, «La mise en cause des autorités administratives...", art. cité, p. 363.

75. C. civ, art. 1382. Ces dispositions posent les bases de la responsabilité civile au Cameroun. 
et le préjudice résulté de la faute. Comme tout type de préjudice, pour être réparable, le préjudice matériel doit être direct ${ }^{76}$, actuel ${ }^{77}$ et certain ${ }^{78}$. En plus, le préjudice à réparer doit être licite ${ }^{79}$. Il convient tout de même de relever que le principe de la réparation du préjudice matériel obéit en droit camerounais à des règles strictes. Selon un adage juridique bien connu, «ne sera réparé que le préjudice, tout le préjudice et rien que le préjudice ". Le principe est celui de stricte équivalence entre la réparation et le dommage. Selon la Cour de cassation, «le propre de la responsabilité civile est de rétablir aussi exactement que possible l'équilibre détruit par le dommage, et de replacer la victime dans la situation où elle se serait trouvée si l'acte dommageable ne s'était pas produit $^{80}$ ». En d'autres termes, il s'agit pour le juge, autant que faire se peut, de rétablir la victime dans une situation identique à celle qui aurait été la sienne si l'acte dommageable ne s'était pas produit tout en évitant qu'elle puisse tirer de la réparation un enrichissement injuste. Le juge ne tiendra compte au moment d'ordonner la réparation que des éléments objectifs fournis. L'estimation du préjudice matériel devra donc être solide, bien présentée, chiffrée et étayée au moyen de pièces justificatives authentiques. Dans tous les cas, défendre ses intérêts est un droit de l'homme reconnu en droit camerounais.

Toutefois, la question s'est posée de savoir si l'auteur de l'acte dommageable pouvait être partiellement exonéré de sa responsabilité civile lorsque la victime n'a pris aucune mesure pour limiter son dommage. Concernant les actes administratifs et plus précisément la voie de fait administrative, le juge judiciaire camerounais, à notre sens, ne s'est pas encore prononcé. Mais une décision de la Cour de cassation française fait état d'une exonération partielle de responsabilité au cas où la victime n'a pris aucune disposition pour limiter les conséquences

76. Direct, le préjudice doit être personnellement subi. Il existe toutefois un principe de "préjudice par ricochet». Classiquement, on prend le cas d'une concubine qui demande réparation du préjudice matériel résultant de la mort de son partenaire qui était le seul du couple à percevoir des rémunérations (Cass. ch. mixte, 27 févr. 1970, JCP 70, II).

77. Actuel, le préjudice doit exister au moment de la demande en réparation.

78. La jurisprudence établit comme certains les types de préjudices suivants : le principe de perte de chance qui consiste en la disparition actuelle et certaine d'une éventualité favorable (Cass. civ., 21 nov. 2006, Jurisdata $\mathrm{n}^{\circ}$ 2006-036009); le préjudice futur qui peut être pris en compte s'il résulte de la prolongation certaine et directe d'un état de choses actuelles.

79. Cass. civ., 24 janv. 2002, grands arrêts, $n^{\circ} 188$.

80. Cass. $2^{\mathrm{e}}$ civ., 28 oct. 1954 , JCP, 1995 , II, 8765. 
de l'acte ${ }^{81}$. À ce niveau, l'évaluation du préjudice matériel devient compliquée puisqu'on ne sait à quel pourcentage le juge va exonérer. Face à cette situation, on tente de faire une appréciation subjective, laquelle caractérise généralement la réparation du préjudice moral.

\subsubsection{La condamnation à la réparation du préjudice moral}

Le préjudice moral est une souffrance subie par une personne par le fait d'un tiers. Il correspond à l'atteinte aux sentiments de la victime, à l'instar de celle qu'elle peut éprouver dans son honneur, dans sa réputation ou dans son affection. Au sens large, le préjudice moral regroupe l'ensemble des atteintes qui n'affectent pas directement un patrimoine ${ }^{82}$. La notion est abstraite et insaisissable au premier abord. Suivant la théorie traditionnelle, le fondement de la responsabilité est la faute commise par une personne douée de raison et de discernement.

Le dommage moral dont il est question ici est la résultante d'un préjudice principal pour lequel on sollicite sa réparation. Certes, une controverse est observée au regard de la réparation du préjudice moral en argent, car le but de la réparation est de rétablir la victime dans la situation où elle était placée antérieurement. Or, l'argent ne répond sûrement pas à ce but et ne peut pas effacer les douleurs antérieures. Les troubles psychologiques ne peuvent donc pas se monnayer. Pour de Page, «la législation qui établit le principe de la compensation en

81. Cass. $2^{\mathrm{e}}$ civ., 26 mars 2015, pourvoi $\mathrm{n}^{\mathrm{o}} 14-16011$.

82. Pour définir le dommage moral, Lalou part d'une notion fondamentale à savoir la notion de droit et oppose le dommage moral au dommage matériel en se basant sur la grande division des droits: les droits patrimoniaux et extrapatrimoniaux. Pour cet auteur, "un dommage est une atteinte à un droit». "Or, il existe deux variétés de droits » : les droits patrimoniaux, lesquels aboutissent à procurer à leurs titulaires des satisfactions pécuniaires ou tout au moins appréciables en argent, comme des droits réels, les droits intellectuels, les bénéfices résultant de l'exercice d'une profession; les droits extrapatrimoniaux comme les droits politiques, les droits inhérents à la personnalité (droit à la vie, à la liberté de conscience ou de parole) et les droits de famille résultant de la qualité d'époux, de parents, alliés (Henri Lalou, Traité pratique de la responsabilité civile, Paris, Dalloz, 1955, p. 105). La distinction du dommage moral et du dommage matériel correspond à cette grande division des droits. C'est cette idée qui a poussé Mazeaud et Tunc à définir cette notion d'une manière lapidaire en disant que le préjudice matériel était le préjudice patrimonial, le préjudice moral, le préjudice extrapatrimonial, non économique (Henri et Léon Mazeaud et André Tunc, Traité théorique et pratique de la responsabilité civile délictuelle et contractuelle, Paris, Montchrestien, 1970 cités par Arsène Kiriza Mashali, La réparation du préjudice moral en droit congolais, mémoire de licence en droit, Université de Kisangani, 2004-2005, p. 17). 
argent du préjudice moral inspire dans l'esprit des citoyens des motifs immoraux ». "Il faut être pénétré d'un profond mépris de la personne d'un homme pour lui suggérer que l'argent soit capable de donner une compensation aux souffrances morales de toute sorte. La transformation d'un préjudice moral en argent est le résultat de l'esprit bourgeois qui apprécie tout en argent, qui considère que tout est à vendre ${ }^{83}$.» Pour les défenseurs de cette thèse, le préjudice moral ne peut être réparé ${ }^{84}$. Le juge se trouve donc dans l'impossibilité d'évaluer le juste prix du préjudice moral.

Cependant, à côté de ces négateurs, il y a des défenseurs de la réparation du préjudice moral. Certes, la réparation ici n'est possible que dans la mesure où le préjudice moral a une incidence matérielle. En matière de voie de fait, par exemple, elle ne vise pas forcément la parfaite reconstruction du passé, mais la réparation du tort psychologique causé par un acte administratif dénaturé. Ainsi, la thèse de la réparation du préjudice moral, à laquelle nous adhérons, peut être défendue. Réparer, ce n'est sûrement pas remettre en état égal à celui qui existait avant l'acte dommageable; ce n'est pas toujours refaire ce qu'on a détruit. Réparer, c'est le plus souvent donner à la victime la possibilité de se procurer des satisfactions équivalentes à ce qu'elle a perdu. Le véritable rôle de la réparation est satisfactoire puisqu'à la faveur de son caractère compensatoire, la réparation procure un effet psychologique certain sur la victime du préjudice. Toutefois, les défenseurs de la thèse ne doivent pas ignorer le danger qui continue à exister quand on marchande l'honneur ou les troubles psychologiques. Cette critique les a conduits à rechercher un autre fondement: l'indemnité allouée n'a plus un caractère compensatoire, mais elle est considérée comme une peine privée. Elle sert plus à l'affirmation d'un droit qu'à compenser un dommage.

Le dernier argument qui a, sur le plan juridique, un coefficient élevé de pertinence, à notre avis, est tiré de l'article 1382 du Code civil. Étant devant le juge judiciaire, ces dispositions trouvent valablement leur raison d'être. En effet, cet article parle de la réparation du dommage tout court. Il ne fait pas de distinction entre le dommage matériel et le dommage moral. Ce serait violer le texte que de distinguer là où le législateur n'a pas distingué. Limiter le sens du terme «dommage»

83. Henri de Page, Traité élémentaire de droit civil belge, Bruxelles, Bruylant, 1964, p. 954.

84. Voir Eugène Trebutien, Cours élémentaire de droit criminel, Paris, A. Durand, t. 1, 1854, p. 17 et suiv.; Henri et Léon Mazeaud et André Tunc, op. cit., p. 387. 
au seul dommage matériel est arbitraire à notre avis. Cette position a pour mérite d'affirmer qu'à côté des droits patrimoniaux, il existe également des droits extrapatrimoniaux, non économiques dont la violation mériterait bien une sanction, c'est-à-dire une réparation. Les dispositions du Code Napoléon applicables au Cameroun n'excluent pas le dommage moral. En outre, l'équité exige que celui qui a causé un dommage ou a posé un acte fautif puisse répondre des conséquences de son acte.

Le droit camerounais admet ainsi le principe de la réparation du préjudice moral. Le juge administratif camerounais l'a d'ailleurs admis dans plusieurs espèces ${ }^{85}$. Certes, il n'y a pas de commune mesure entre l'argent et la souffrance; mais la réparation, bien qu'imparfaite, reste possible. Les dommages-intérêts alloués en pareil cas revêtent un caractère compensatoire. Tout en admettant la réparation du dommage moral, le droit camerounais ne nie pas la difficulté qu'il a à établir une équivalence entre la souffrance et l'argent. En la matière, l'appréciation du juge est souveraine. C'est lui qui, en fonction des circonstances et des preuves administrées à l'audience, fixe le quantum des dommagesintérêts.

Comme on peut le constater, les juridictions ne sont tenues par aucun barème, et c'est là la difficulté d'évaluation du préjudice. En plus, les juridictions ne sont pas tenues de s'expliquer sur le choix des critères d'évaluation retenus ou de suivre les parties dans le détail de leur argumentation. Elles peuvent souverainement apprécier l'existence et l'étendue des préjudices ainsi que le montant des indemnités propres à en assurer l'entière réparation. Ainsi, en matière de réparation du préjudice moral né de la voie de fait, le juge a une liberté d'appréciation dictée par sa conviction et sa conscience.

La réparation peut être par nature ou par équivalence. Elle peut aussi être symbolique. Pour être réparé, le dommage moral doit être direct, actuel, certain et consister dans la violation d'un intérêt légitime. Le propre de la responsabilité civile ou administrative est de rétablir aussi

85. Dans l'affaire Nkondock Émile Valentin c/ État du Cameroun (CS/CA, jugement $\mathrm{n}^{\mathrm{o}} 72,26$ mai 1983), l'État du Cameroun a été condamné à payer 20000 ooo F CFA de préjudice moral au recourant. Dans l'espèce Tameghi Boniface (AMSECOM, AMSECONCOM) c/ État du Cameroun (CS/CA, jugement $n^{\circ}$ 50/84-85, $1^{\text {er }}$ févr. 1985), ce dernier a été condamné à payer à la partie demanderesse à titre de réparation du préjudice moral la somme de 92000 ooo F CFA. Dans l'affaire Nkongo Bidzanga Maximilien c/ État du Cameroun (MFPRA) (CS/CA, jugement $n^{\circ} 92 / 2008 / C A / C S, 13$ août 2008), la partie défenderesse a été condamnée à payer au recourant à titre de réparation du préjudice moral la somme de 5 ooo ooo F CFA. 
exactement que possible l'équilibre détruit par le dommage et de replacer la victime dans la situation où elle se serait trouvée si l'acte dommageable ne s'était pas produit ${ }^{86}$.

Qu'on soit en présence du préjudice matériel ou moral, l'administration peut être condamnée par le juge judiciaire en cas de voie de fait à le réparer. Nul n'ignore que la fonction des indemnités allouées dans ce cas est, en l'occurrence, non une réparation du dommage, c'est-à-dire un rétablissement de l'état antérieur à celui-ci, mais une compensation pécuniaire à l'atteinte qui a été irrémédiablement portée à cet état. Pour le juge, la seule difficulté peut résulter au niveau de la fixation du quantum de cette compensation pour qu'elle soit reconnue satisfactoire.

Que ce soit le préjudice matériel ou moral, la réparation se fait devant le juge du fond. Si la demande excède dix millions de francs CFA, c'est le tribunal de grande instance qui est compétent en l'affaire. Si, par contre, la demande est inférieure ou égale à dix millions de francs CFA, c'est le tribunal de première instance qui est compétent ${ }^{87}$.

$\mathrm{Au}$ Cameroun, l'examen de la jurisprudence relève que la majorité des justiciables, en raison de l'urgence, font généralement appel au juge des référés. Or, ce juge se limite seulement à constater, qualifier et ordonner la cessation de la voie de fait. Les justiciables, dans l'immense majorité, ignorent qu'après le juge des référés, on peut aller devant le juge du fond solliciter réparation. Ainsi, ils doivent désormais savoir qu’après que le juge des référés s'est prononcé, la partie ayant intérêt peut saisir le juge du fond, en fonction du quantum de la demande, pour obtenir réparation.

Lorsque l'administration devient rebelle au droit, en agissant hors la loi, l'unique solution crédible dans un État de droit est le recours au juge ${ }^{88}$. Ce recours au juge s'illustre bien en cas de voie de fait

86. Cass. $2^{\mathrm{e}}$ civ., 7 déc. $1978 ; 9$ juil. $1981 ; 4$ févr. $1982 ; 13$ janv. 1988.

87. L. $\mathrm{n}^{\mathrm{o}} 2011 / 027,14$ déc. 2011 , art. 15 , al. 1 (b) et art. 18 , al. 1 (b) modifiant et complétant certaines dispositions de L. $\mathrm{n}^{\mathrm{o}}$ 2006/015, 29 déc. 2006 portant organisation judiciaire.

88. . Selon une définition plus ancienne, l'État de droit est un système institutionnel dans lequel la puissance publique est soumise au droit. Le juriste autrichien Hans Kelsen a défini cette notion d'origine allemande au début du $\mathrm{xx}^{\mathrm{e}}$ siècle, comme un «État dans lequel les normes juridiques sont hiérarchisées de telle sorte que la puissance (publique) s'en trouve limitée». De nos jours, l'État de droit est une situation juridique dans laquelle chacun est soumis au respect du droit, du plus simple individu jusqu'à la puissance publique. Il est très étroitement lié au respect de la hiérarchie des normes, de la séparation des pouvoirs et des droits fondamentaux. Il reste aussi étroitement lié au développement du constitutionnalisme. Sur cette notion, lire Hans Kelsen, Théorie pure du droit, Paris, Dalloz, 1962, p. 411 et suiv; Raymond Carré de Malberg, Contribution à la théorie générale de l'État, Paris, 
administrative. En examinant de nombreux cas tranchés par le juge judiciaire, on constate que l'occasion a été offerte à ce dernier d'attirer l'attention de l'administration sur les limites de ses pouvoirs. Ce juge, dans le contentieux de la voie de fait administrative, a eu le mérite de réaffirmer son audace à travers l'étendue de ses compétences. Il a aussi saisi l'opportunité donnée par les justiciables pour rappeler l'étendue de ses compétences dans un contentieux de la voie de fait administrative. Ainsi, au Cameroun, le juge judiciaire se trouve habilité, en matière de contentieux de la voie de fait administrative, à constater les actes de l'administration, les qualifier de voie de fait ou non, ordonner toute mesure pour qu'il y soit mis fin et réparer le préjudice causé par lesdits actes. Sous cet angle, l'administration se trouve déchue du privilège de juridiction et déposséder de ses prérogatives de puissance publique.

Chargé de cours de droit public, HDR Université de Dschang, Cameroun

Recueil Sirey, 1920-1922; Jacques Chevalier, «L'État de droit», RDP 1988, p. 316 et suiv.; L'État de droit, Paris, Montchrestien, 1999, p. 53 et suiv; Léo Hamon, "L'État de droit et son essence», RFDC 1990, p. 699 et suiv; Jacques Chevallier, "La mondialisation de l'État de droit », dans Mélanges Philippe Ardant, Paris, LGDJ, 1999, p. 325-337 ; Benjamin Boumakani, «L'État de droit en Afrique centrale", dans Vers une société de droit en Afrique centrale, 1970-2000, colloque de Yaoundé, 14-16 novembre 2000, p. 87-109; Léopold Donfack Sokeng, "L'État de droit en Afrique ", Afrique Juridique et Politique, vol. 1, $\mathrm{n}^{\circ}$ 2, 2002, p. 87-125; Champlain Dounkeng Zele, "L'État de droit face aux défis de son effectivité en Afrique», Annales de la FSJP de l'Université de Dschang, $\mathrm{n}^{\circ}$ 8, 2004, p. 189-214. 